\title{
Effect of shelter porosity on downwind flow characteristics
}

\author{
Š. Nosek ${ }^{1, a}$, R. Kellnerová1 ${ }^{\text {, K. Jurčáková }{ }^{1}, \text { Z. Jaňour }}{ }^{1}$, H. Chaloupecká ${ }^{1,2}$, and M. Jakubcová ${ }^{1,3}$ \\ 1 Institute of Thermomechanics AS CR, Dolejškova 1402/5, Prague, Czech Republic \\ 2 Charles University in Prague, Faculty of Mathematics and Physics, Department of Meteorology and Environment \\ Protection, V Holešovičkách 2, Prague, Czech Republic
}

3 Czech University of Life Sciences Prague, Faculty of Environmental Sciences, Department of Water Resources and Environmental Modeling, Kamýcká 1176, Prague, Czech Republic

\begin{abstract}
Previous wind-tunnel studies were focused mainly on lonely standing windbreaks or wind fences with respect to their wind velocity reduction efficiency and effective shelter distance. In presented wind-tunnel study, we investigated the effects of a three different fence porosities $(0.5,0.25$ and 0$)$ embodied in a shelter-like building for coal convey by means of two-component Laser Doppler Anemometry (LDA). The turbulent flow characteristics behind the fences were compared with those performed without the fence. For characterization of the fence effectiveness we used following quantities: wind-speed and turbulence kinetic energy reduction, and time fractions of the turbulent coherent structures associated with the sediment transport (sweeps and outward interactions). Results from mentioned quantities revealed that for the case of embodied fence the shelter construction has significant impact on the flow characteristics behind. The fence of the 0.5 porosity has been indicated as the most shelter effective considering the studied quantities.
\end{abstract}

\section{Introduction}

The windbreaks or wind fences have been studied since the 1940th. The main objectives of these studies remain the same: shelter efficiency and the factors that help to optimize the windbreak or wind fence design with respect to control the particle sedimentation (sand, dust, snow, etc.). The fence shelter efficiency is evaluated as the reduction of the wind velocity and downwind distance for which wind velocity remains below the threshold velocity [1]. Here the threshold velocity relates to the particle movement initiation. Previous studies (e.g. [2]) observed that the shelter efficiency is mainly influenced by the fence geometry and porosity. As one might expected, lower porosity brings about higher wind velocity reduction. However, higher turbulent intensities, shear stresses and pressures are produced as well. These flow turbulent characteristics are unfavourable to fence efficiency since decreasing the fence shelter distance and enhancing the particle uplift just downstream the fence.

In this paper, we present twofold extension relating to the fence shelter efficiency analysis: construction complexity and turbulent coherent structures. Previous wind-tunnel studies were mainly focused on a lonely standing windbreaks or wind fences. However, there are many practical fence applications. Thus, in present study, we considered the fence integration into the shelter for avoiding the coal dust escape from an convey belt to the surrounding area. This will give a better insight into a practical fence utilization.

The second fence shelter efficiency analysis extension follows the experimental study on aeolian sediment trans-

\footnotetext{
a e-mail: nosek@it.cas.cz
}

port over a sand dune [9], which revealed that the turbulent coherent structures, sweeps and outward interactions, have a dominant influence on sand transport. This finding is in contrast to research in fluvial domain where turbulent structures associated with the bursting processes, sweeps and ejections, were found to be dominant for sediment transport [3].

\section{Experimental setup}

The experiment was carried out in the low-speed Environmental wind-tunnel of the Institute of Thermomechanics of the Czech academy of sciences in Nový Knín. For detailed wind-tunnel description see [4]. The schema of the shelter model for convey belt of scale 1:100 positioned at the wind-tunnel test section with respect to the wind-tunnel coordinates is presented in figure 1a. Detail of the model, where the measures are normalised by the shelter model characteristic height $H=90 \mathrm{~mm}(H=9 \mathrm{~m}$ at full scale), is presented in figure $1 \mathrm{~b}$. The fence, made of polypropylene fibres, was positioned at the leeward side of the shelter at $x / H=0$ and $z / H=0.17$ and runs along the entire lateral shelter leeward side (from $y / H=-8.3$ to $y / H=8.3$ ). The gap that arisen between the bottom of the wind tunnel and the fence is necessary for dust explosiveness prevention. We investigated the following fence porosities $\epsilon=0,0.25$ and 0.5 , defined according to [5] as

$$
\epsilon=\left(\frac{B-D}{D}\right)^{2}
$$

where $B$ is the fence opening width and $D$ is the fence fibre diameter. 
(a)
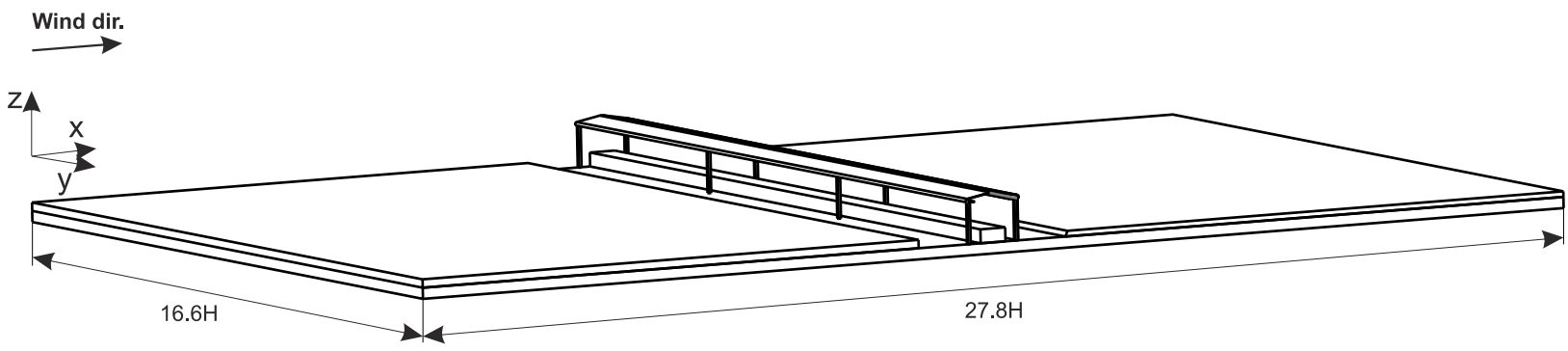

(b)

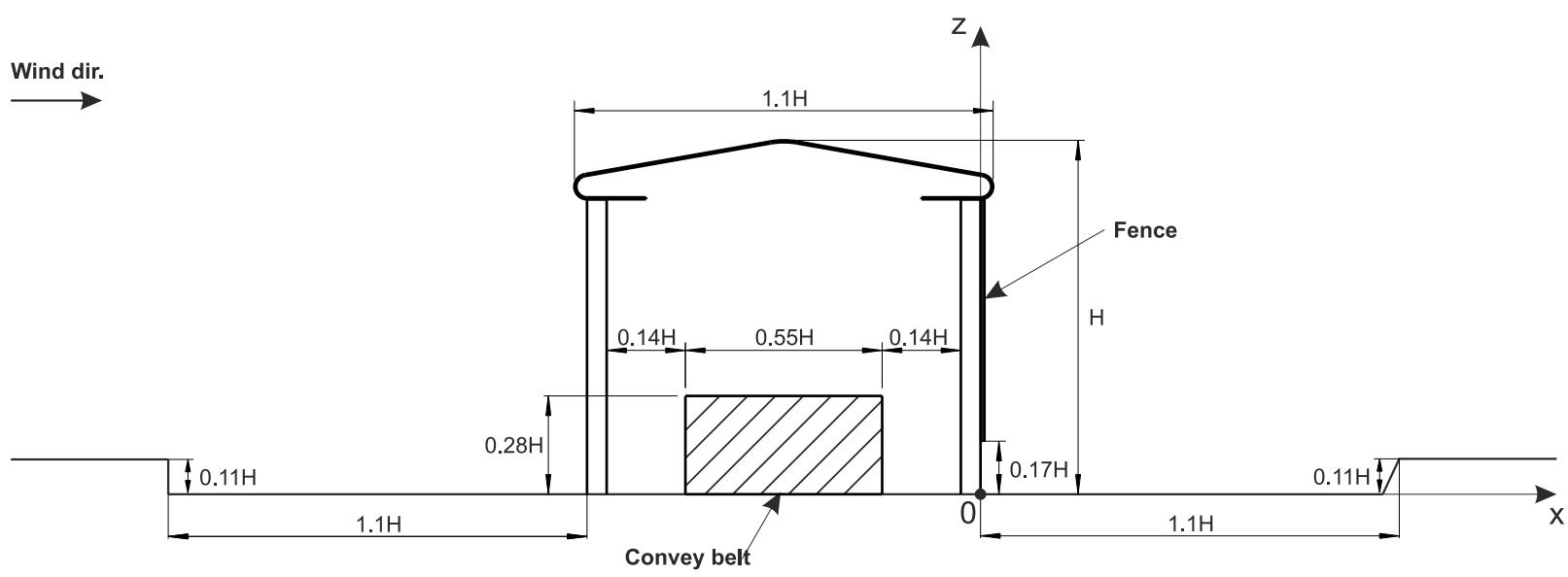

Fig. 1: Schemas of the shelter model with respect to the wind-tunnel coordinates $x, y$ and $z$ : (a) isometric view of the whole model incorporated in the wind-tunnel measuring section; (b) detailed side view with the origin of the coordinate system for the measurement grid (the origin of the $y$ coordinate is in the middle of the model lateral direction, 16.6H/2).

The wind-tunnel free stream velocity was maintained at $6 \mathrm{~m} \mathrm{~s}^{-1}$ during the whole experiment. For the characteristic height of the shelter model the Reynolds number was sufficiently high $(R e=34600)$ to fulfill the Reynolds number independence $\left(R e_{\text {crit }}=11000\right)$, recommended by [6]. The free stream velocity also fulfil the criterium for the fence porosity independence on pressure drop according to [7].

The turbulent boundary layer simulating the moderately rough atmospheric boundary layer for the area of the interest (e.g. moderate hills, shrubs) was developed along the $20 \mathrm{~m}$ long wind-tunnel development section. Four vortex generators were spaced laterally at the beginning of the development section and the remaining part was covered by the roughness elements. The boundary layer characteristics were measured at the model entrance with the twodimensional Laser Doppler Anemometry (LDA) based on DANTEC BSA F-60 burst processor. The parameters of the longitudinal velocity profile (roughness length $z_{0}$, displacement length $d_{0}$ and friction velocity $u_{*}$ ) were derived from the logarithmic law and are compared with those recommended by the VDI guidelines [8] in table 1 . The parameters of the longitudinal velocity profile as well as the longitudinal and vertical turbulent profiles (not shown here) were in accordance with the VDI guidelines [8].

\section{Data Analysis}

The data were collected from the measured grid of the vertical plane $x z$ downstream from the fence by LDA system at a rate ranging from 1 to $2 \mathrm{kHz}$. The sampling time for
Table 1: Approaching boundary layer parameters for moderately rough terrain

\begin{tabular}{cccc}
\hline & $z_{0}(\mathrm{~m})$ & $d_{0}(\mathrm{~m})$ & $u_{*}\left(\mathrm{~m} \mathrm{~s}^{-1}\right)$ \\
\hline $\operatorname{Exp}$ & 0.15 & 0 & 0.17 \\
VDI & $0.12-0.18$ & 0 & - \\
\hline
\end{tabular}

each point of the measured grid was kept constant at $90 \mathrm{~s}$. The measurement error was established on $0.83 \%$ from 36 measured reference velocities, $U_{\text {ref }}$, obtained during the experiment from the same point at dimensionless height $z / H=5.5$.

The origin of the coordinate system for the measurement grid is depicted in figure $1 \mathrm{~b}$, where the scheme of the vertical cut at $y=0$ is presented. We measured at 6 heights 13 points downstream from the fence for each fence porosity and without the fence. One extra height $(z / H=0.1)$, consisting of 5 downstream points, was measured to provide the data below the fence gap $(z / H<0.17$, see figure 1b) for each model type as well.

For analysing the fence effectiveness, we used a dimensionless wind-speed reduction coefficient according to [2]

$$
R_{U}=1-\frac{U_{\operatorname{dim}}}{U_{\operatorname{dim} 0}}
$$

where $U_{\text {dim }}=U / U_{\text {ref }}$ is the dimensionless mean velocity measured at a given point and fence porosity and $U_{\operatorname{dim} 0}=U_{0} / U_{\text {ref } 0}$ is the dimensionless mean velocity measured at the same given point without the fence. The same 


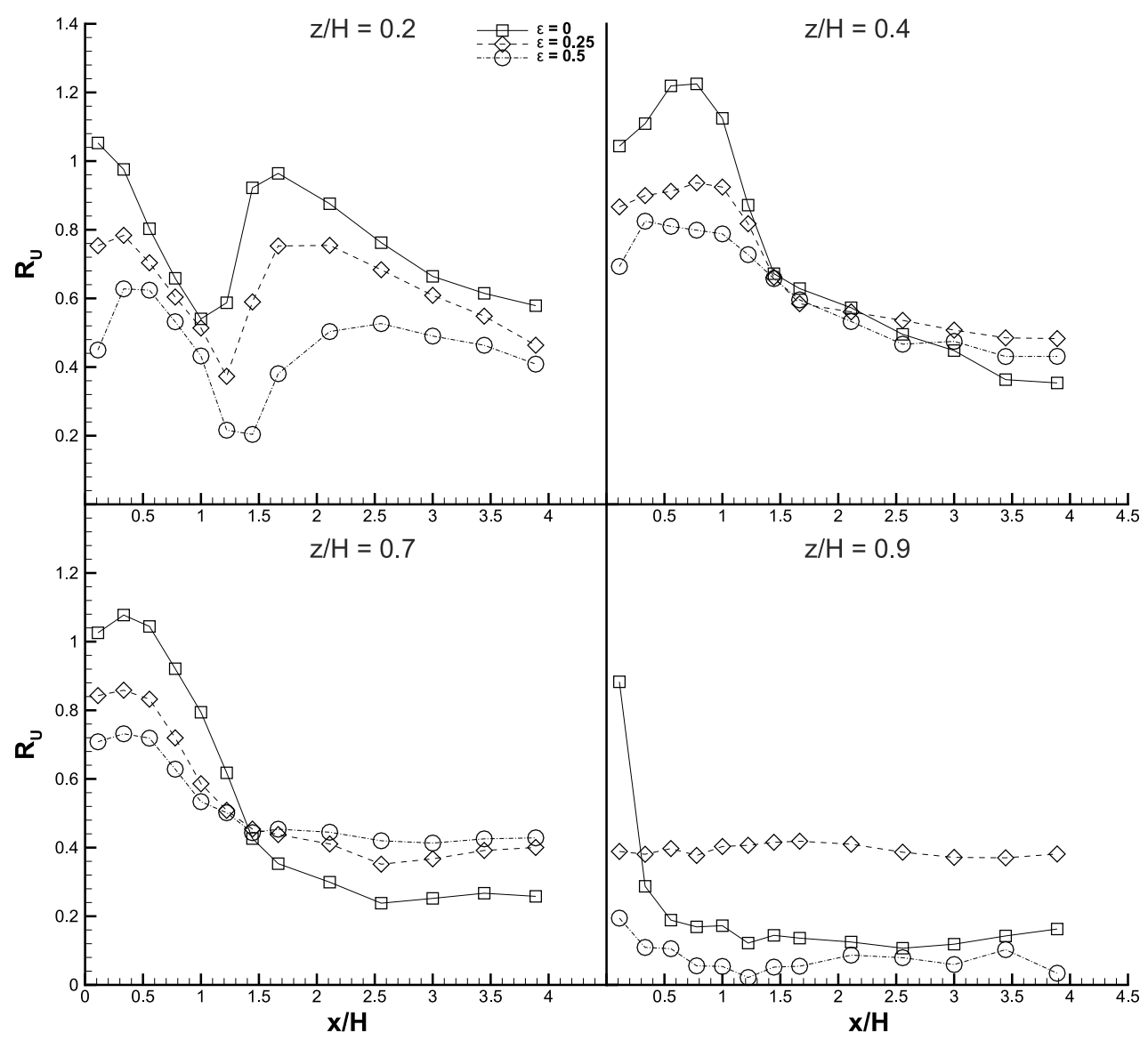

Fig. 2: Dimensionless longitudinal velocity reduction coefficient of the fence with porosity $\epsilon=0$ (squares), $\epsilon=0.25$ (diamonds) and $\epsilon=0.5$ (circles) as a function of the dimensionless streamwise distance from the fence, $x / H$, for four different dimensionless heights $z / H=0.2,0.4,0.7$ and 0.9 .

was applied for the dimensionless turbulent kinetic energy reduction coefficient

$$
R_{T K E}=1-\frac{T K E_{\operatorname{dim}}}{T K E_{\operatorname{dim} 0}} .
$$

Here, we assumed that the turbulent flow is a quasi twodimensional and used the following formulas for dimensionless turbulent kinetic energies

$$
T K E_{\text {dim }}=\frac{\left\langle u^{\prime 2}\right\rangle+\left\langle w^{\prime 2}\right\rangle}{2 U_{r e f}^{2}}, \quad T K E_{\text {dim } 0}=\frac{\left\langle u_{0}{ }^{2}\right\rangle+\left\langle w_{0}{ }^{2}\right\rangle}{2 U_{r e f 0}^{2}},
$$

where $u^{\prime}$ and $w^{\prime}$ is the fluctuation of the longitudinal and vertical velocity, respectively, \langle\rangle is the time averaging and the zero index denotes the case without the fence. If $R_{U}$ and $R_{T K E}$ are equal to one, the fence reduces the windspeed and turbulent kinetic energy to zero and is $100 \%$ efficient. The negative values mean that the fence is increasing the wind-speed and turbulent kinetic energy, respectively.

Assuming the same approach for the sand transport [9], we performed the quadrant analysis according to [10] in order to identify turbulent coherent structures. This conditioning technique separates the flow into four discrete categories according to four quadrants of the scatter plots of two turbulent quantities, $u^{\prime}$ and $w^{\prime}: 1$. outward interactions $\left(u^{\prime}>0, w^{\prime}>0\right), 2$. sweeps $\left(u^{\prime}>0, w^{\prime}<0\right)$, 3. inward interactions $\left(u^{\prime}<0, w^{\prime}<0\right)$ and 4. ejections $\left(u^{\prime}<0\right.$, $\left.w^{\prime}>0\right)$. We used the hole quadrant analysis in order to filter insignificant events such that the attribution of the flow structure occurred if $\left|u^{\prime} w^{\prime}\right|>\sigma_{u w}$, where $\sigma_{u w}$ is the standard deviation of the kinematic stress $u^{\prime} w^{\prime}$ and represents the selected "hole threshold".

We analysed the fence porosity efficiency with respect to relevant events for sediment transport (sweeps and outward interactions) by means of their time fraction to the total sampling time $T$ defined by [11] as

$$
D_{i}=\frac{1}{T} \int_{0}^{T} I_{i}(t) \mathrm{d} t
$$

where $D_{2}$ and $D_{1}$ is total sweep and outward interaction durations within $T$, respectively, and $I_{i}$ is the indicator function, where $I_{i}=1$, if $u^{\prime} w^{\prime}$ is within quadrant $i=1,2$ and $I_{i}=0$, otherwise.

\section{Results}

\subsection{Reduction coefficients}

The dimensionless longitudinal velocity reduction coefficient, $R_{U}$, of three fence porosities as a function of the dimensionless streamwise distance from the fence, $x / H$, is presented in figure 2 . The four graphs of $R_{U}$ are plotted for dimensionless height $z / H=0.2,0.4,0.7$ and 0.9 , respectively.

As one expected, the solid wall, $\epsilon=0$ (square symbols in figure 2), has the highest wind-speed reduction. 


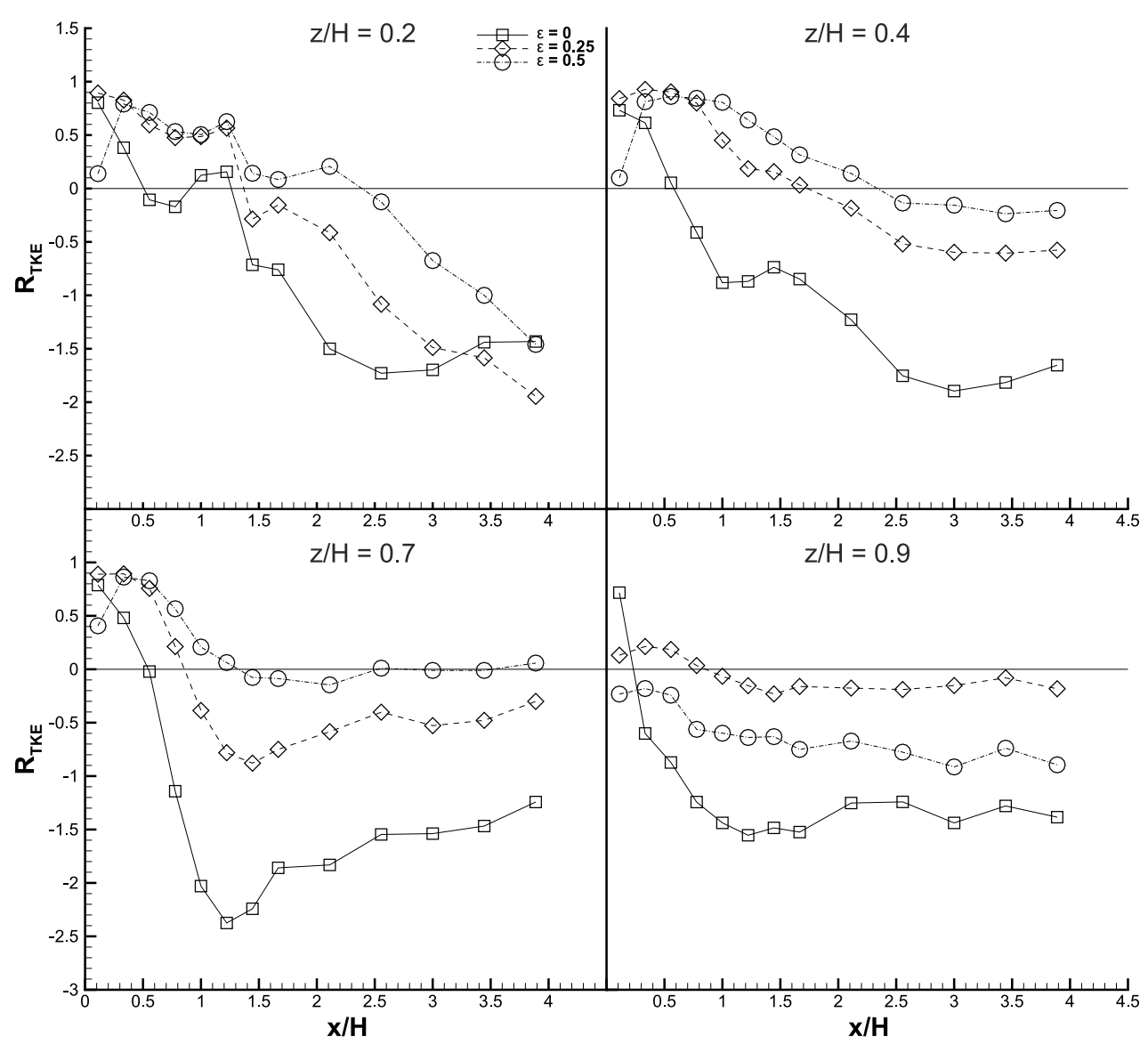

Fig. 3: The same as in figure 2 but for the dimensionless turbulent kinetic energy reduction coefficient.

(a)

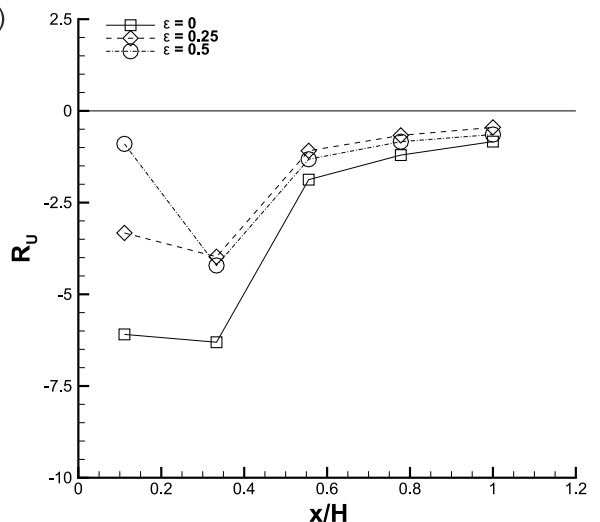

(b)

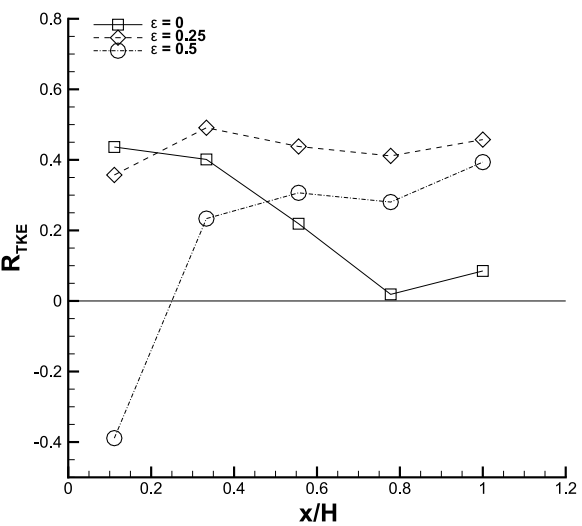

Fig. 4: Reduction coefficient of the (a) dimensionless longitudinal velocity and (b) dimensionless turbulent kinetic energy for the fence porosity $\epsilon=0$ (squares), $\epsilon=0.25$ (diamonds) and $\epsilon=0.5$ (circles) as a function of the dimensionless streamwise distance from the fence, $x / H$, at dimensionless height $z / H=0.1$.

However, this reduction is absolute (for all investigated heights) only at the fence vicinity $(x / H=0.1)$. For heights $z / H>0.4$ we can observe that $R_{U}$ decreases with the increasing downstream distance and height. The $R_{U}$ is even lower than the $R_{U}$ of the fence with the highest porosity $(\epsilon=0.5)$ for $x / H>1.5$ and $z / H>0.2$. The values of $R_{U}$ in case of the solid wall means that the backward wind direction (vortex) is present. The wind-speed reduction is independent on the dimensionless downstream distance $x / H$ from ceratin value of $x / H$. We can observe that the higher the height the shorter the downstream distance is for the wind-speed reduction independence on downstream distance.

Another parameter for determining the fence effectiveness, the dimensionless turbulent kinetic energy reduction coefficient $R_{T K E}$, is presented in figure 3 . The meanings of the symbols and coordinates are the same as in figure 2 .

From $R_{T K E}$ point of view, the solid wall is even the worst case with respect to the sediment transport behind the shelter. It increases the turbulent kinetic energy (negative values of $R_{T K E}$ ) at downstream distances further than $x / H=0.5$ at all investigated heights, compared to the case without the fence. Both investigated porous fences 


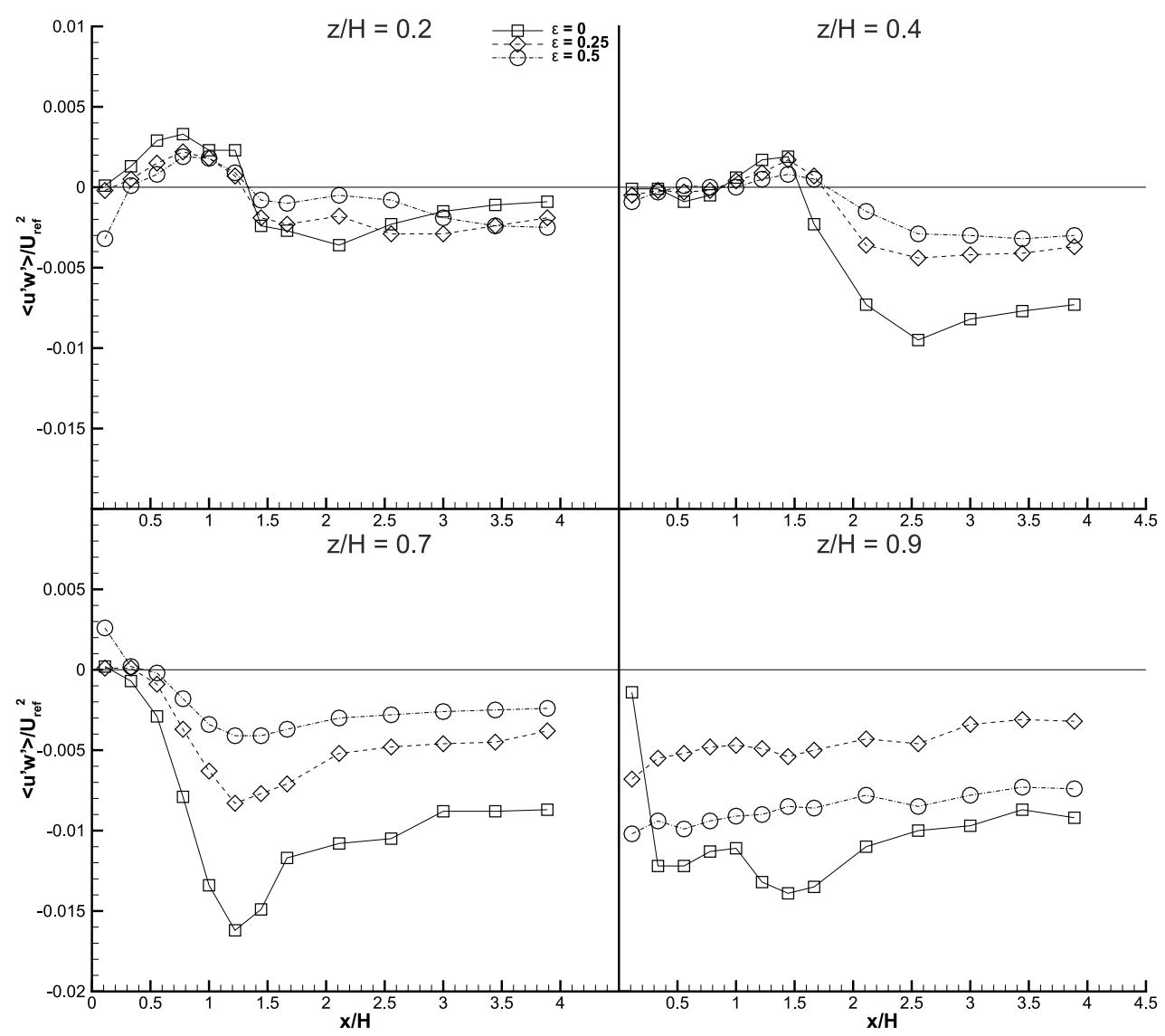

Fig. 5: The same as in figure 2 but for the dimensionless vertical momentum flux.

are more efficient compared to the solid wall. The best results of $R_{T K E}$ are achieved with the fence porosity of $\epsilon=0.5$ (circles in figure 3), where up to the downstream distance of $x / H=2$ and for the lower investigated heights $(z / H=0.2$ and 0.4$)$ the fence still reducing the TKE, compared with the case without the fence. At the vicinity of the fence $(x / H<1)$, the $T K E$ reductions of the fence with 0.5 porosity are very similar with those from the fence of 0.25 porosity (diamonds in figure 3 ).

If we observe the effect of a necessary vertical gap between the fence and the bottom of the model $(z / H=0.1)$, we can see from the figure $4 \mathrm{a}$ and figure $4 \mathrm{~b}$ that strong wind-speed accelerations and not so strong turbulent kinetic energy reductions occur for all investigated porosities, respectively. Thus, the simulated vertical gap has unfavourable effect for sediment transport.

Because of better wind-speed and turbulent kinetic energy reductions are attained by fence of 0.25 and 0.5 porosity, respectively, the most favorable porosity cannot be stated. Hence, turbulent coherent structure analysis at following chapter would give a better insight to the fence porosity effectiveness.

\subsection{Turbulent coherent structures analysis}

Because the presented turbulent coherent structures are the partitions of the total vertical momentum flux $\left\langle u^{\prime} w^{\prime}\right\rangle$, we present the dimensionless momentum flux $\left\langle u^{\prime} w^{\prime}\right\rangle / U_{r e f}^{2}$ in figure 5 as well. As in previous presented results, the vertical momentum flux is plotted as a function of dimension- less streamwise distance $x / H$ at four graphs for dimensionless heights $z / H=0.2,0.4,0.7$ and 0.9 , respectively.

The effect of the gap is evident at the height $z / H=$ 0.2 and up to downstream distance from the fence $x / H=$ 1.5 , where upward momentum flux occurs. At the middle height $(z / H=0.4)$, the fence momentum flux reduction effect $\left(\left\langle u^{\prime} w^{\prime}\right\rangle / U_{r e f}^{2} \approx 0\right)$ up to $x / H=1.5$ can be observed. For higher levels, the strong momentum flux downwards are present from downstream distances even close to the fence $(x / H>0.5)$. The lowest values of the momentum flux are attained by the fence of the 0.5 porosity across the downstream distance and up to $z / H=0.7$.

The time fractions of the relevant coherent structures, sweeps $\left(D_{2}\right)$ and outward interactions $\left(D_{1}\right)$, with respect to the dimensionless streamwise distance $x / H$ and height $z / H$ are presented in figure 6 . Using the hole quadrant analysis that filters the "weaker" coherent structures brings about the maximum time fraction decay approx. to 0.3 of the total momentum flux (approx. 70\% of the events are filtered, not shown here). Thus, the maximum time fraction value of the sweep or outward interaction might be 0.3 from all investigated points.

The strong impact of the height on sweep time fractions can be observed from figure 6 ( $D_{2}$, filled symbols). At the levels higher than the middle height of the fence $z / H>0.4)$ and at the downstream distances further than one height of the fence $(x / H>1)$, the sweep time fractions dominates over outward interaction ones. The highest values of the sweep time fractions (around 10\% of sampling time) are attained by solid wall (filled squares). If we consider that the time fractions are filtered from the 


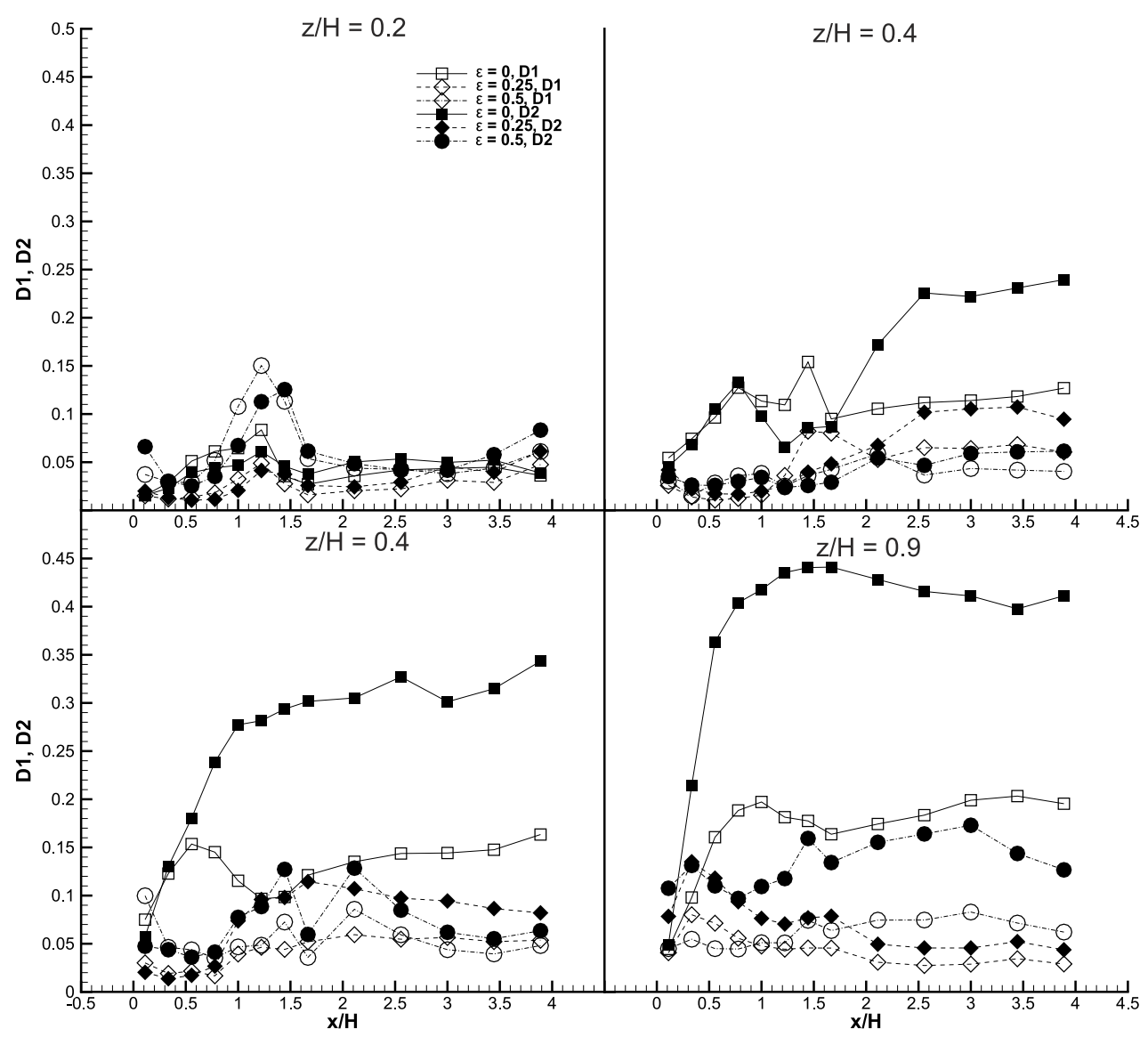

Fig. 6: The same as in figure 2 but for the sweep ( $D_{2}$, filled symbols) and outward interaction $\left(D_{1}\right.$, empty symbols $)$ time fractions.

(a)

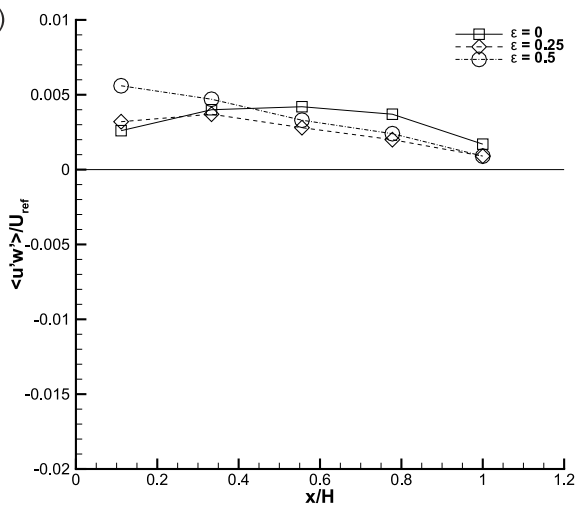

(b)

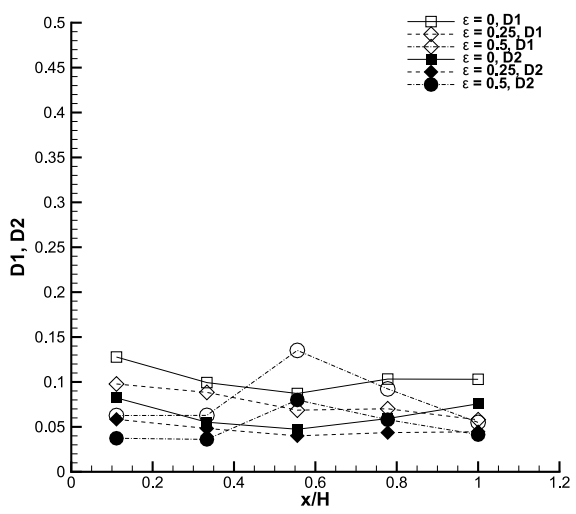

Fig. 7: (a) Dimensionless vertical momentum flux and (b) sweep ( $D_{2}$, filled symbols) and outward interaction $\left(D_{1}\right.$, empty symbols) time fractions for the fence porosity $\epsilon=0$ (squares), $\epsilon=0.25$ (diamonds) and $\epsilon=0.5$ (circles) as a function of the dimensionless streamwise distance from the fence, $x / H$, at dimensionless height $z / H=0.1$.

insignificant ones, the dominant sweep time fractions occupy approx. $30 \%$ of the relative sampling time at these higher levels. In comparison with the sweep time fractions for the fences of the 0.5 and 0.25 porosities, this represents approx. four time higher occupancy of the relative sampling time. The lowest values of the sweep time fractions between can be observed for the fences of 0.25 porosities (filled diamonds) at all investigated heights.

The outward interaction time fractions $\left(D_{1}\right.$, empty symbols in figure 6) attained the highest values for the solid wall at height $z / H<0.9$. For porous fences, the highest values can be observed the fence vicinity $(x / H<1.5)$ and lower heights $(z / H<0.4)$. This points out on the ef- fect of the vertical gap below the fences. As for the case of the sweeps, the lowest values of the outward interaction time durations are attained by the fence of 0.25 porosity. However, these values are slightly lower than in case of the fence with 0.5 porosity. Compared with the sweeps, lesser impact of the height on outward interaction time fraction can be observed.

From figure $7 \mathrm{a}$ and figure $7 \mathrm{~b}$ we can see no significant effect of the fence porosity on total momentum flux and coherent structure time fractions in the region close to the vertical gap $(z / H=0.1)$, respectively. In the case of the coherent structure time fractions the outward interactions 
(empty symbols in figure 7b) are more frequent than the sweep events (filled symbols in figure 7b).

\section{Conclusions}

The presented wind-tunnel experiment on porous shelter model revealed the impact of the geometry and porosity on downstream flow quantities. We demonstrated that not only the wind-speed and turbulent kinetic reduction coefficients but also the vertical momentum flux and its partitions should be taken into account for the shelter efficiency determination with respect to the aeolian sediment transport. According to these turbulent quantities the non-porous shelter shows the most unfavourable flow for the sediment transport, hence the worse shelter efficiency. The necessary shelter construction arrangement, comprising the vertical gap below the shelter porous/non-porous leeward wall, has unfavourable effect on shelter efficiency of all investigated shelter porosities. The best shelter performance associated with the highest turbulent kinetic reduction, the lowest total vertical momentum fluxes, and very low dominant turbulent coherent structures time fractions were observed for the shelter of 50\% porosity. This porosity was worse than others only for the wind-speed reduction coefficient and have slightly lower values of the dominant coherent structures time fractions than the fence of $25 \%$ porosity. These results for the very high fence porosity suggest that the vertical gap at the shelter leeward wall would be unnecessary considering the dust explosiveness limits, hence improve sheltering effect.

\section{Acknowledgements}

This work was supported by the Czech Science Foundation GACR (project GAP15-18964S) and by the institutional support RVO: 61388998.

\section{References}

1. Z. Dong, W. Luo, G. Qian, P. Lu, H. Wang, Journal of Arid Environments 74, (2010) 193-207

2. W. M. Cornelis, D. Gabriels, Journal of Arid Environments 61, (2005), 315-332

3. W. Yue, C. L. Lin, V. C. Patel, J. Fluid Eng. 127, (2005), 858-864

4. L. Kukačka, Š. Nosek, R. Kellnerová, K. Jurčáková, Z. Jaňour, TheScientificWorldJournal 2012, (2012) 13

5. Z. Jaňour, P. Jonáš, Strojnický časopis 42, (1991) 443450 (in Czech)

6. W. H. Snyder, Guidelines for fluid modelling of atmospheric diffusion (EPA office of Air quality, USA, 1981) 199

7. P. Jonáš, Čs. Čas. Fys. 7, (1957) 76-86 (in Czech)

8. VDI, Verein Deutcher Ingenieure, GER, 200036

9. G. F. S. Wiggs, C. M. Weaver, Geophysical research letters 39, (2012)

10. S. S. Lu, W. W. Willmarth, Earth Sci. Rev. 80, (1973)

11. G. Katul, D. Poggi, D. Cava, J. Finnigan, BoundaryLayer Meteorology, 120, (2006) 367-375 